\title{
Electric characteristics of pseudo-spark during breakdown phase
}

\author{
Bin Lin and Quantie Chow \\ Institute of Mechanics, Chinese Academy of Sciences, Beijing 100080, People's \\ Republic of China
}

Received 3 November 1992

\begin{abstract}
An analysis of the time-dependent resistive voltage and power deposition during the breakdown phase of pseudo-spark is presented. The voltage and current were measured by specially designed low-inductance capacitive voltage divider and current measuring resistor. The measured waveforms of voltage and current are digitized and processed by a computer program to remove the inductive component, so as to obtain resistive voltage and power deposition. The influence of pressure, cathode geometry and charging voltage of storage capacitors on the electrical properties in the breakdown phase are investigated. The results suggest that the breakdown phase of pseudo-spark consists of three stages. The first stage is mainly hollow cathode discharge. In the second stage, field-enhanced thermionic emission takes place, resulting in a fast voltage drop and sharp rise of discharge current. The third stage of discharge depends simply on the parameters of the discharge circuit.
\end{abstract}

\section{Introduction}

Pseudo-spark is a low-pressure pulsed discharge with special electrode geometry. The pseudo-spark chamber consists of two axially symmetric plate electrodes with central holes. The typical gas pressure and separation of electrodes are $0.075-0.75$ Torr and several millimetres respectively $[1,2]$. The discharge in such a chamber is similar to the ordinary spark discharge at higher pressure with regard to its high current and extremely rapid current rise, even though it is a glow discharge instead of an arc [2]. The properties of glow with rapid current rise suggest that pseudo-spark is suitable for application in high-power closing switches. The experimental results for pseudo-spark switches and back-lighted thyratrons (thyratron-type pseudo-spark switches) show their merits, namely that they have current rise rate and life time better than that of the thyratron, while their life time and jitter are better than that of the spark gap, with the other electrical characteristics being comparable [3]. Another application of pseudo-spark is as a kind of electron and ion beam generator $[2,4]$.

The discharge behaviour of pseudo-spark has been studied by many researchers; however, the timedependent characteristics such as resistive voltage, current and power deposition were not investigated carefully, though these characteristics give important information for the operation of a pseudo-spark switch and for theoretical analysis of essential processes in the pseudo-spark discharge.
In order to derive the power deposition in the discharge gap to estimate the temperature rise, it is necessary to know the time-dependent resistive voltage and current. Though the current measurement is quite easy by means of a current-measuring resistor or Rogowski coil, accurate measurement of the timedependent resistive voltage is rather difficult because of the fast breakdown of pseudo-spark and the temporal variation of the inductive component of the gap voltage. In this paper, the time-dependent voltage of pseudospark is measured accurately by a specially designed low-inductance capacitive voltage divider. The inductive component of the measured voltage is further removed by a computer program with digitized experimental voltage and current waveforms, to obtain the resistive voltage and power deposition. Experiments were carried out for different pressures and charging voltages with the aperture diameters and cathode cage diameters from 2 to $5 \mathrm{~mm}$ and from 5 to $18 \mathrm{~mm}$ respectively. The mechanism of pseudo-spark in the breakdown phase is discussed in terms of the time-dependent electrical characteristics.

\section{Experimental set-up}

Figure 1 gives a schematic diagram of the experimental apparatus. The pseudo-spark between two electrodes at separation $5 \mathrm{~mm}$ is triggered by charge injection [2]. The discharge circuit is arranged axially to reduce loop inductance, which is composed of 33 ceramic storage 


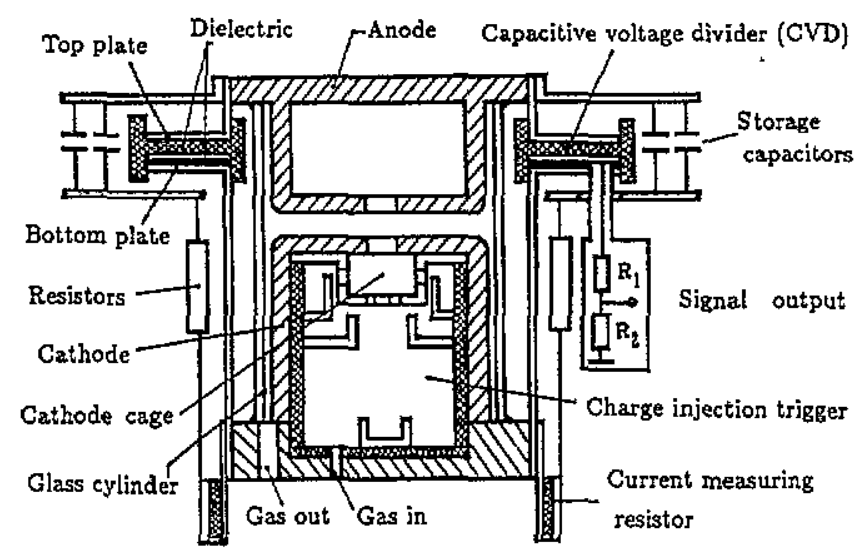

Figure 1. Schematic experimental set-up and electrical circuit.

capacitors ( $30 \mathrm{kV}, 3300 \mathrm{pf}$ each) and 10 parallel ceramic resistors with combined resistance $1 \Omega$ to achieve a unipolar pulse. The annular arrangement of the lowinductance capacitive voltage divider (CVD) around the pseudo-spark chamber is also shown in figure 1. The capacitors $C_{1}$ and $C_{2}$ of the CVD are composed of three flat rings, the upper and middle rings form capacitor $C_{1}$ while the middle and lower rings form $C_{2}$. The thicknesses of dielectric polytetrafluoroethylene of $C_{1}$ and $C_{2}$ are 5 and $0.05 \mathrm{~mm}$, and their capacitances are approximately 50 and $5000 \mathrm{pF}$ respectively. The CVD is directly connected to the anode and cathode by two cylinders coaxial with the pseudo-spark electrodes. This geometry minimizes the inductance of the CVD. The rise time of the CVD is theoretically estimated to be $1 \mathrm{~ns}$ and experimentally measured to be much less than $5 \mathrm{~ns}$ [5].

The pseudo-spark current is measured by a cylindrical current-measuring resistor, also with a calculated rise time less than $1 \mathrm{~ns}$ [5]. The simuitaneous voltage and current waves are recorded by a Tektronix 7844 high-speed oscilloscope.

\section{Data processing technique}

There is a deviation of output signal from the actual voltage waveform due to voltage decay across $C_{2}$. So the values of $C_{1}, C_{2}, R_{1}$ and $R_{2}$ are chosen to ensure that voltage decay during the measurement period is as small as possible, that is the time constant $\left(R_{1}+\right.$ $\left.R_{2}\right) C_{2}$ should be large in comparison with the pulse length; but the time constant $\left(R_{1}+R_{2}\right) C_{2}$ is limited by the demand for fast rise time of the resistive voltage divider. This deviation can be removed by analysing the equivalent circuit of the CVD and discharge circuit as shown in figure 2 . The voltage $u_{1}(t)$ of the pseudospark chamber can be expressed by

$$
\begin{aligned}
u_{1}(t) & =\frac{\left(C_{1}+C_{2}\right)\left(R_{1}+R_{2}\right)}{C_{1} R_{2}} u_{2}(t) \\
& +\frac{1}{R_{2} C_{1}} \int_{0}^{t} u_{2}(t) \mathrm{d} t+u_{1}(0) \\
& -\frac{\left(C_{1}+C_{2}\right)\left(R_{1}+R_{2}\right)}{C_{1} R_{2}} u_{2}(0)
\end{aligned}
$$

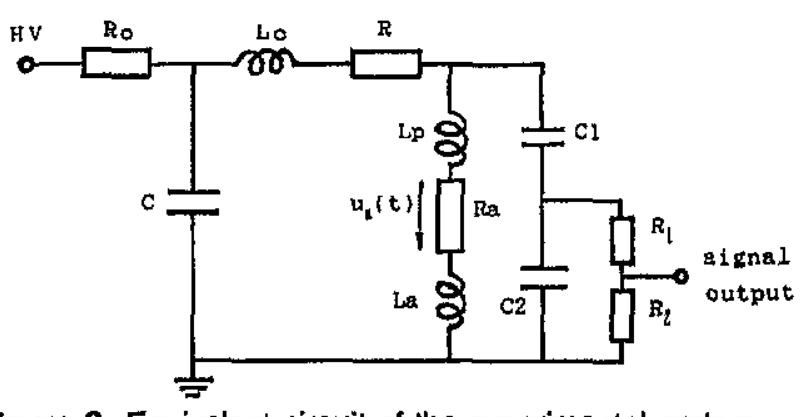

Figure 2. Equivalent circuit of the experimental system.

where $u_{2}(t)$ is the output signal. In our case $u_{1}(0)$ equals the charging voltage of the storage capacitor and $u_{2}(0)$ is zero.

In order to deduce the power deposition during discharge, it is necessary to know the resistive voltage $u_{2}(t)$ across the pseudo-spark gap. The power deposition is simply

$$
P(t)=u_{2}(t) i(t) .
$$

The measured voltage $u_{1}(t)$ of the whole pseudospark chamber, which consists of the inductive voltage of the electrodes, the inductive voltage of the discharge column and the resistive voltage across the gap, can be written as

$$
u_{1}(t)=i(t) u_{\mathrm{a}}(t)+\left[L_{\mathrm{a}}(t)+L_{\mathrm{p}}\right] \frac{\mathrm{d} i}{\mathrm{~d} t}+i(t) \frac{\mathrm{d} L_{\mathrm{a}}(t)}{\mathrm{d} t} .
$$

In this experiment the inductance of electrodes $L_{\mathrm{p}}$ is $9.28 \mathrm{nH}$ [5], and the time-dependent inductance of the pseudo-spark channel $L_{\mathrm{a}}(t)$ is determined by the temporal variation of the spark diameter. According to the streak camera photographs of the pseudo-spark column given in $[6,7]$, we suggest that the diameter of the pseudo-spark column increases with a constant rate, reaches its maximum at the time of maximum current, and then remains constant. The diameter of the molten area remaining on the cathode surface after discharge can be taken as the maximum diameter of the column [7]. Assuming that current flows uniformly through the pseudo-spark column, the value of $L_{\mathrm{a}}(t)$ is given approximately by

$$
L_{\mathbf{2}}(t)=2 s \ln \left(\frac{D}{\phi_{\mathbf{2}}(t)}\right)
$$

where $s$ is the separation of electrodes $(5 \mathrm{~mm}), D$ is the effective diameter of the current return path and $\phi_{\mathrm{a}}(t)$ is the time-dependent diameter of the pseudo-spark column. The measured voltage and current waves are digitized. The resistive voltage $u_{\mathrm{a}}(t)$ and power deposition $P(t)$ can be calculated from equations (1)-(4) by a computer program, in which the current waveform is numerically differentiated to provide $\mathrm{d} i(t) / \mathrm{d} t$ by a cubic spline function.

\section{Experimental results and discussion}

The voltage and current are measured for different gas pressures of helium, charging voltage and geometry of 


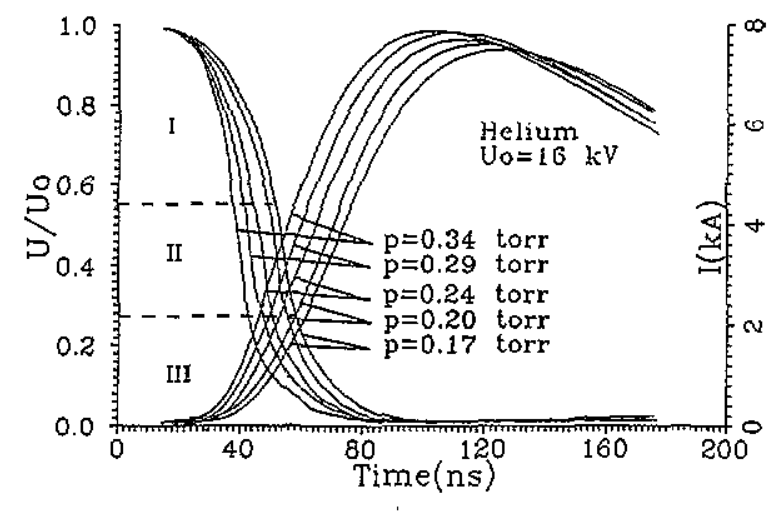

Figure 3. Time-dependent resistive voltage and current for different pressures.

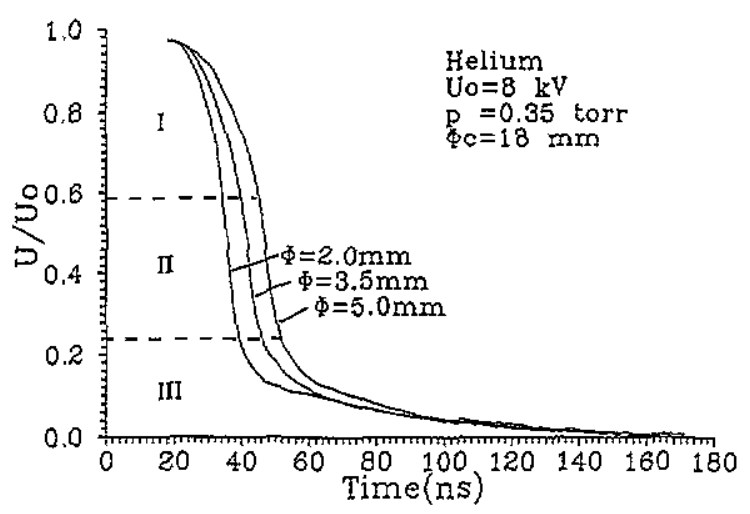

Figure 4. Time-dependent resistive voltage for different aperture diameters.

cathode. The evacuated chamber at $10^{-3}$ Torr is filled with $\mathrm{He}$ of purity $99.99 \%$ to wash twice, then kept at a certain pressure of pure helium. A series of discharges is repeated twice per second for several minutes to clean the surface of electrodes and the interior of chamber. The time-dependent resistive voltages and discharge currents for pressures from 0.17 to 0.34 Torr are shown in figure 3. The voltage breakdown intervals (from $90 \%$ $U_{0}$ to $10 \% U_{0}$ ) in a range of 29-37 $\mathrm{ns}$, increase with decreasing pressure. Figure 4 shows the time-dependent resistive voltage for a cathode with aperture diameters of $2.0,3.5$ and $5.0 \mathrm{~mm}$. Figure 5 shows the timedependent resistive voltage for cathode cage diameters of 5,10 and $18 \mathrm{~mm}$. The curves in figures 4 and 5 show that the breakdown intervals shorten with decrease in aperture diameter or increase in cathode cage diameter.

The pseudo-spark is initiated by a transient hollow cathode discharge on the back of the cathode $[6,8,9]$. The small cathode aperture allows a limited electric field to penetrate into the hollow cathode cage so that the collisions and secondary processes can take place more efficiently than in the main gap. The hollow cathode effect leads to a significant rise of current under two distinct mechanisms [9]. The first is an effect of 'Pendel electrons' [3], namely the electrons inside the cathode cage reflect back and forth between the cathode sheaths of the hollow cathode and effectively ionize the plasma. Another mechanism is simply due to the confining effect of the small cathode aperture.

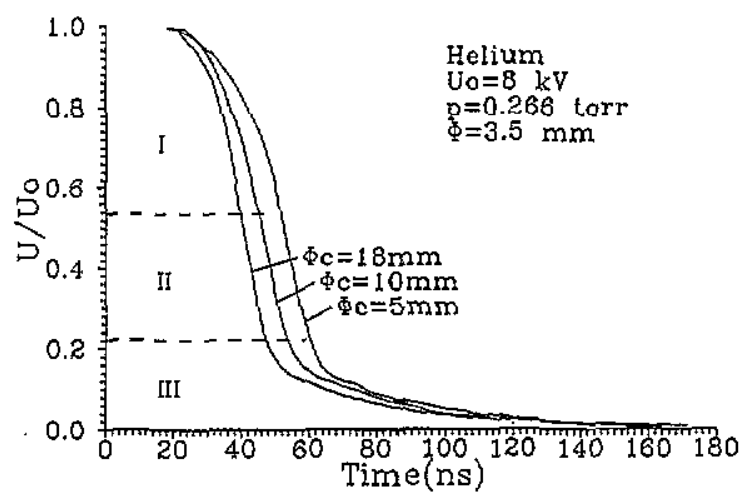

Figure 5. Time-dependent resistive voltage for different cathode cage diameters.

When the cathode sheath thickness, which decreases as discharge grows, becomes much smaller than the dimension of the hollow cathode, most of the cathode cage volume will be full of plasma, the secondary electrons from the sheath may not be accelerated and the confining effect plays a more important role for current rise. When the sheath thickness decreases to the order of $1 \mu \mathrm{m}$, the high electric field on the cathode surface and the high temperature of the cathode, heated by the impacts of ions in the former stage, can lead to field-enhanced thermionic emission $[10,11]$. This emission may be responsible for the extremely fast drop of the gap voltage and sharp rise of current.

In figures 3-5 the breakdown interval of the pseudospark appears to consist of three stages. In stage I, the gap voltage drops with increasing rate. In stage II, the voltage drops with nearly constant (fastest) rate. In stage III, the voltage drop rate decreases until the gap voltage vanishes. It can be seen that the gas pressure, the cathode aperture and the cathode cage can only influence stage I of breakdown. These phenomena indicated that stage $I$ is mainly hollow cathode discharge. The influence of gas pressure and the diameters of cathode aperture and cathode cage on the rate of voltage drop can be explained as follows. The pseudo-spark works on the left-hand side of the Paschen curve, so the higher pressure (lower value of $E / N$ ) results in more ionizations by electron impacts in the cathode region. Increasing the diameter of the cathode cage leads to longer free paths for electrons travelling inside the cathode cage and higher ionization in the hollow cathode discharge stage. When the aperture diameter varies from 5 to $2 \mathrm{~mm}$, the voltage drop becomes faster for smaller diameter in stage I simply due to the confining effect of hollow cathode.

The power deposition in the pseudo-spark gap as a function of time is shown in figure 6, which is deduced from figure 3. Comparing figure 3 with figure 6 , we can see that the occurrence of maxima on the power deposition curves coincides with the beginning of stage II. The peak density of power deposition is (13-17) $\times 10^{6} \mathrm{~W} \mathrm{~cm}^{-2}$ on a cathode area of approximately $0.6 \mathrm{~cm}^{2}$ [5] with a half peak width 32-35 ns.

According to the analysis by Hartmann et al [11], an average power density of $(10-20) \times 10^{6} \mathrm{~W} \mathrm{~cm}^{-2}$ 


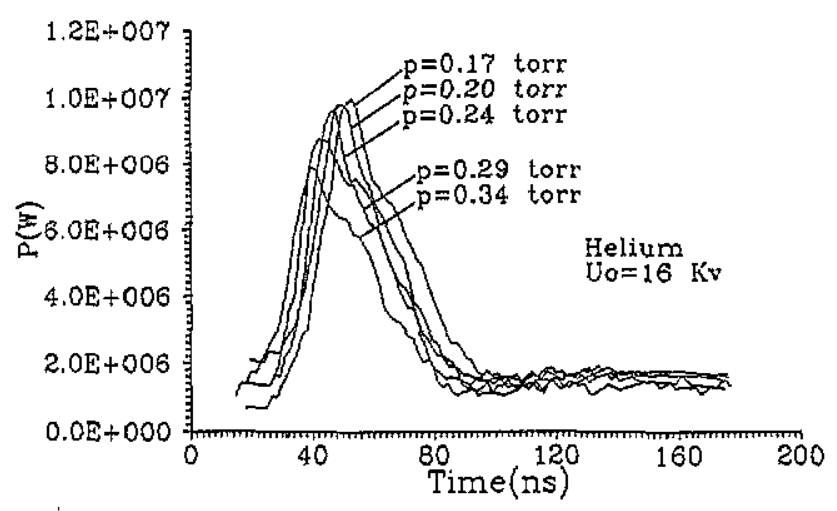

Figure 6. Power deposition as a function of time for different pressures.

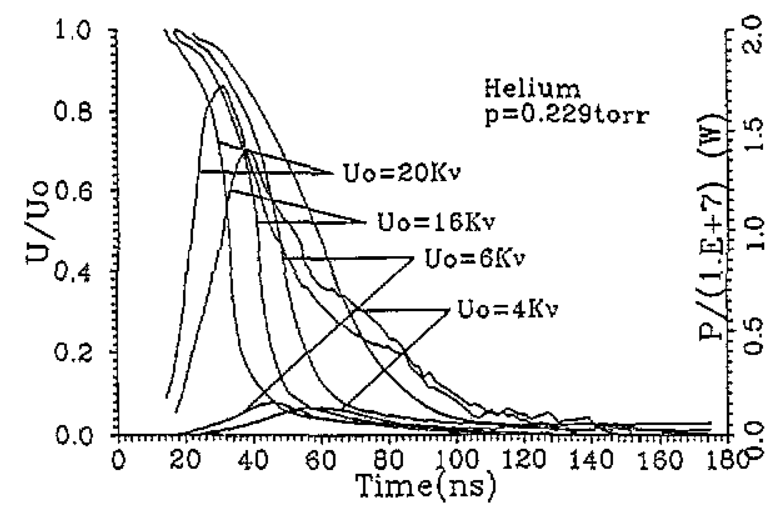

Figure 7. Influence of charge voltage on resistive voltage and power deposition.

lasting $30 \mathrm{~ns}$ can heat a thin layer on the cathode up to about $3000 \mathrm{~K}$, producing field-enhanced thermionic emission. So figures 6 and 3 demonstrate that the rapid drop of voltage in stage II is a result of ficld-enhanced thermionic emission on the cathode surface.

Figure 7 shows the influence of charging voltage $U_{0}$ on the voltage drop and power deposition of the pseudo-spark. The voltage drops much more slowly for $U_{0}=6 \mathrm{kV}$ and $U_{0}=4 \mathrm{kV}$ than for higher $U_{0}$. Since the peak values of power deposition in figure 7 are relatively small for $U_{0}=4 \mathrm{kV}$ and $U_{0}=6 \mathrm{kV}$, so field-enhanced thermionic emission cannot take place and the voltage drop rates in figure 7 are low.

In stage III, the impedance of the discharge gap is much smaller than that of the discharge circuit, so the discharge current depends mainly on the parameters of the discharge circuit.

\section{Conclusion}

The pseudo-spark voltage and current were measured by a low-inductance capacitive voltage divider and current-measuring resistor. The time-dependent resistive voltage and power deposition were calculated.
The results suggest that the breakdown phase of the pseudo-spark consists of three stages. In the first stage the dependence of the voltage drop rate on gas pressure, cathode aperture and cathode cage size can be explained by two hollow cathode effects: the "Pendel electron' effect and the confining effect, in qualitative agreement with previous numerical simulation $[9,12]$. The mechanism for second stage discharge is fieldenhanced thermionic emission, which corresponds to the fast drop of gap voltage and sharp rise of current. The high peak value of power deposition at the beginning of stage II is evidence for field-enhanced thermionic emission, which is in quantitative agreement with the analysis by Hartmann et al [11]. Our results demonstrate that, for the switching application, the high breakdown speed of the pseudo-spark in the second stage due to thermionic emission is independent of gas pressure, cathode aperture and cathode cage size.

\section{References}

[1] Bloess D, Kamber I and Riege H 1983 The triggered pseudospark chamber as a fast switch and as a high-intensity beam source Nucl Instrum Methods $205173-84$

[2] Mechtersheimer $G$, Kohler R, Lasser T and Meyer $R 1986$ High repetition rate, fast current rise, pseudospark switch J. Phys. E: Sci. Instrum. 19 466-70

[3] Frank $\mathrm{K}$ et al $1988 \mathrm{High}-$ power pseudospark and BLT switches IEEE Trans. Plasma Sci. 16 371-23

[4] Boggasch E and Rhee M J 1990 High-brightness pseudospark produced electron beam Appl. Phys. Lett. 56 1746-8

[5] Lin Bin 1991 Characteristics of pseudospark discharge and its switches $P h D$ Thesis Institute of Mechanics, Chinese Academy of Sciences

[6] Choi P, Chuaqui $\mathrm{H}$ et al 1989 Plasma formation in a pseudospark discharge IEEE Trans. Plasma Sci 17 $170-3$

[7] Hartmann W, Dominic V, Kirkman G F et al 1988 Evidence for large area super emission into a high-current glow discharge Appl Phys. Lett. 53 1699-701

[8] Frank K and Christiansen J 1989 The fundamentals of the pseudospark and its applications IEEE Trans. Plasma Sci. 17 248-53

[9] Boeuf J P and Pitchford L C 1991 Pseudospark discharge via computer simulation IEEE Trans. Plasma Sci. $19286-96$

[10] Elartmann W and Gundersen M A 1988 Origin of anomalous emission in superdense glow discharge Phys. Rev. Lett. 60 2371-4

[11] Hartmann W, Dominic V, Kirkman $G$ and Gundersen M 1989 An analysis of the anomalous high-current cathode emission in pseudospark and back-of-theCathode lighted thyratron switches J. Appl. Phys. 65 4388-94

[12] Hoyoung Pak and Kushner Mark J 1990 Multibeam-bulk model for electron transport during commutation in an optically triggered pseudospark thyratron Appl. Phys. Lett. 57 1619-21 